\title{
Redispersible Spray-Dried Powder Containing Nanoencapsulated Curcumin: the Drying Process Does Not Affect Neuroprotection In vitro
}

\author{
Diego Fontana de Andrade, ${ }^{1}$ Branko Vukosavljevic, ${ }^{2}$ Juliana Bender Hoppe, ${ }^{3}$ Adriana Raffin Pohlmann,, 4 \\ Sílvia Stanisçuaski Guterres, ${ }^{1}$ Maike Windbergs, ${ }^{2,5}$ Irene Külkamp-Guerreiro, ${ }^{1}$ \\ Christianne Gazzana Salbego, ${ }^{3}$ and Ruy Carlos Ruver Beck ${ }^{1,6,7}$
}

Received 10 May 2019; accepted 3 August 2019; published online 12 August 2019

\begin{abstract}
A redispersible spray-dried formulation containing curcumin-loaded, lipid-core nanocapsules (LNC-C) was developed for oral administration. The neuroprotective activity of curcumin after the spray-drying process was evaluated in vitro. The spray-dried powder (SD-LNC-C) was produced using a drying adjuvant composed of a blend of maltodextrin and L-leucine (90:10 w/w). Acceptable process yield $(\sim 70 \%)$ and drug content $\left(6.5 \pm 0.2 \mathrm{mg} \mathrm{g}^{-1}\right)$ were obtained. SD-LNC-C was formed by smooth, spherical-shaped particles, and confocal Raman analysis indicated the distribution of the LNC-C on the surface of the leucine/ maltodextrin agglomerates. The surface of the agglomerates was formed by a combination of LNC-C and adjuvants, and laser diffraction showed that SD-LNC-C had adequate aqueous redispersion, with no loss of controlled drug release behaviour of LNC-C. The in vitro curcumin activity against the lipopolysaccharide (LPS)-induced proinflammatory response in organotypic hippocampal slice cultures was evaluated. Both formulations (LNC-C and SDLNC-C) reduced TNF- $\alpha$ to similar levels. Therefore, neuroprotection of curcumin in vitro may be improved by nanoencapsulation followed by spray-drying, with no loss of this superior performance. Hence, the redispersible spray-dried powder proposed here represents a suitable approach for the development of innovative nanomedicines containing curcumin for the prevention/treatment of neurodegenerative diseases.
\end{abstract}

KEY WORDS: curcumin; nanocapsules; neuroinflammation; powders; spray-drying.

Electronic supplementary material The online version of this article (https://doi.org/10.1208/s12249-019-1501-1) contains supplementary material, which is available to authorized users.

\footnotetext{
${ }^{1}$ Programa de Pós-Graduação em Ciências Farmacêuticas, Universidade Federal do Rio Grande do Sul, Porto Alegre, RS, Brazil.

${ }^{2}$ Department of Drug Delivery, Helmholtz Centre for Infection Research (HZI) and Helmholtz Institute for Pharmaceutical Research Saarland (HIPS), Saarbruecken, Germany.

${ }^{3}$ Departamento de Bioquímica, Universidade Federal do Rio Grande do Sul, Porto Alegre, Rio Grande do Sul, Brazil.

${ }^{4}$ Instituto de Química, Universidade Federal do Rio Grande do Sul, Porto Alegre, RS, Brazil.

${ }^{5}$ Institute of Pharmaceutical Technology and Buchmann Institute for Molecular Life Sciences, Goethe University, Frankfurt am Main, Germany.

${ }^{6}$ Departamento de Produção e Controle de Medicamentos, Universidade Federal do Rio Grande do Sul, Avenida Ipiranga, 2952, Porto Alegre, RS 90610-000, Brazil.

${ }^{7}$ To whom correspondence should be addressed. (e-mail: ruy.beck@ufrgs.br)
}

\section{INTRODUCTION}

Curcumin has been commercialized as a flavouring agent, preservative, colouring agent, and food supplement in several countries. It is a polyphenol derived from the root of the rhizome Curcuma longa (turmeric) (1). Because of the wide array of beneficial properties of curcumin, scientific and medical communities are interested in clarifying the pharmacological properties of this polyphenol. Besides its welldescribed antioxidant and anti-inflammatory properties, curcumin is also able to modulate the expression of various molecular targets, such as cytokines, enzymes and receptors, making it a promising molecule for the prevention and treatment of neurodegenerative diseases related to oxidative processes $(2,3)$.

However, the bioavailability of curcumin after oral administration is low, which in turn negatively affects its therapeutic potential (4). Our recent studies suggested the encapsulation of this molecule in lipid-core nanocapsules (LNC) could overcome this drawback (4-7). Briefly, LNC are vesicular structures containing an organogel composed of liquid and solid lipids as the core, which is surrounded by a polymeric wall $(8,9)$. Using this system to encapsulate a drug- 
like curcumin can improve drug stability, modulate the tissue distribution and control the drug release over time, contributing to the results achieved in the studies cited above (1012).

Our recent findings have shown the superior in vitro and in vivo neuroprotective performance of curcumin encapsulated in LNC. In this sense, the high bioavailability of curcumin in the central nervous system (CNS) is an important advantage of its nanoencapsulation. The superior activity of curcumin-loaded LNC was explained by Jacques et al. and Hoppe et al. in models of cognitive impairment induced by cigarette and the peptide $A \beta(1-42)$, respectively (6,7). In fact, the high curcumin bioavailability in the CNS afforded by encapsulation in LNC was reported by Zanotto-Filho et al. in a pre-clinical model of glioma (5).

LNC work as drug shuttles, administered intravenously or orally, which explains their superior performance in the CNS compared with the non-encapsulated drug (13). Considering that the oral route is usually the most convenient, safe and accepted administration pathway, it becomes a promising alternative in the administration of drug-loaded LNC for brain delivery (14).

LNC are produced as promising pharmaceutic liquid formulations; nevertheless, their production as intermediate dry powders could broaden their applications, extending shelf-life, minimizing microbiological contaminations and improving/increasing portability and dosing. Hence, spraydrying has been proposed as a technology to obtain dry powders containing LNC (15-18). Our research group recently showed the recovery of the physicochemical properties of LNC after the redispersion of their spray-dried powders in water, using a blend of hydrophilic adjuvants in the drying process (19).

Therefore, the approach to convert curcumin-loaded LNC aqueous suspensions into a redispersible powder, by spray-drying, as a suitable pharmaceutical dosage form for its oral administration was evaluated in this study. The main objective was to evaluate the impact of the drying process and the subsequent aqueous redispersion on the biological response of curcumin using an in vitro model of neuroinflammation. This novel experimental design represents an original contribution to the field of pharmaceutical research as a prior investigative effort for further in vivo studies, since no previous reports have evaluated the influence of the spray-drying process on the in vitro biological response of powders containing drug-loaded nanocapsules.

\section{MATERIAL AND METHODS}

\section{Materials}

Poly( $\varepsilon$-caprolactone) (Mw $80 \mathrm{~kg} \mathrm{~mol}^{-1}$ ), sorbitan monostearate and curcumin were purchased from SigmaAldrich (São Paulo, Brazil). Grape seed oil and Polysorbate 80 were provided by Delaware (Porto Alegre, Brazil) and Henrifarma (São Paulo, Brazil), respectively. Maltodextrin was obtained from Roquette (Lestrem, France). L-Leucine was purchased from Fagron (São Paulo, Brazil).

\section{Preparation of Curcumin-Loaded Lipid-Core Nanocapsule Suspensions}

Curcumin-loaded lipid-core nanocapsule (LNC-C) suspensions were prepared by nanoprecipitation of a preformed polymer (8). An acetone solution (135 mL), composed of $0.05 \mathrm{~g}$ of curcumin, $825 \mu \mathrm{L}$ of grape seed oil, $0.5 \mathrm{~g}$ of poly $(\varepsilon-$ caprolactone) and $0.19 \mathrm{~g}$ of sorbitan monostearate, was prepared at $40^{\circ} \mathrm{C}$. The acetone solution was then injected under stirring into a polysorbate $80(0.14 \%$, w/v) aqueous solution $(270 \mathrm{~mL})$. After this step, evaporation under reduced pressure at $40^{\circ} \mathrm{C}$ was carried out to remove the organic solvent and to concentrate the dispersion to a final volume of $50 \mathrm{~mL}$. Blank lipid-core nanocapsule (LNC-B) suspensions were prepared following the same protocol, except curcumin was omitted from the formulation. Three LNC-C and LNC-B batches were prepared and protected from light at room temperature.

\section{Physicochemical Characterization of LNC-C Suspensions}

\section{Particle Size and Zeta Potential Analyses}

The mean size and polydispersity index (PDI) of LNC suspensions were assessed by dynamic light scattering (DLS) (Zetasizer Nano ZS®, Malvern Instruments, Malvern, UK) at $25^{\circ} \mathrm{C}$ after diluting an aliquot of the LNC-C formulation in ultrapure water $(1: 500, \mathrm{v} / \mathrm{v})$. Furthermore, LNC-C particle size distribution was evaluated by laser diffraction (LD) (Mastersizer® 2000, Malvern Instruments, Malvern, UK). The zeta potential was measured based on electrophoretic mobility (Zetasizer Nano ZS®, Malvern Instruments, UK), after diluting of an aliquot of the LNC-C suspension in $10 \mathrm{mM} \mathrm{NaCl}$ aqueous solution (1:500, v/v) (8). All the analyses were carried out for 4 independent batches $(n=4)$.

\section{Drug Content Analyses}

The curcumin content was assayed by reversed phase high-performance liquid chromatography (RP-HPLC), as previously described (5). The method was linear between 10.0 and $30.0 \mu \mathrm{g} \mathrm{mL}^{-1} \quad(y=128780.24 x+123333.13, r=$ $0.9999)$, precise (RSD lower than $3.2 \%$ ) and specific. The drug content of each LNC-C batch was assayed after dissolving LNC-C $(1 \mathrm{~mL})$ in acetonitrile $(10 \mathrm{~mL})$. These samples were centrifuged at $4120 \times g$ for $10 \mathrm{~min}$. Next, $2 \mathrm{~mL}$ of the supernatants was collected and diluted in $10 \mathrm{~mL}$ of the mobile phase. Finally, the samples were filtered $(0.45-\mu \mathrm{m}$ PVDF membrane) and immediately analysed by the RPHPLC.

\section{Determination of Encapsulation Efficiency}

To determine the encapsulation efficiency (EE), the ultrafiltration/centrifugation technique was carried out using the Microcon ${ }^{\circledR}$ centrifugal filter (10,000 Da, Merck Millipore, Darmstadt, Germany) at $2150 \times g$ for $10 \mathrm{~min}$. After dilution in acetonitrile, the level of non-encapsulated curcumin in the ultrafiltrate was analysed by the RP-HPLC, according to the method described above. EE (\%) was estimated based on the difference between the total amount of curcumin as measured 
in the LNC-C suspension and the non-encapsulated curcumin concentration assayed in the ultrafiltrate. The percentage of encapsulated curcumin was calculated considering the experimental drug content of curcumin assayed by the RP-HPLC.

\section{Preparation of Spray-Dried Curcumin-Loaded Lipid-Core Nanocapsule Powders}

Employing the process parameters previously described by de Andrade et al. (19), spray-dried curcumin-loaded lipidcore nanocapsule (SD-LNC-C) powders were prepared using a Buchi Mini Spray Dryer B-290 (Buchi, Switzerland), which contained a nozzle with a cap orifice diameter of $0.7 \mathrm{~mm}$. The drying adjuvant, composed of a blend of maltodextrin and Lleucine $(90: 10 \mathrm{w} / \mathrm{w})$, was added to the LNC-C suspension at a final concentration of $5 \%(\mathrm{w} / \mathrm{v})(19)$. Twenty minutes before the drying process, the blend of adjuvants was dissolved, under magnetic stirring, in $25 \mathrm{~mL}$ of ultrapure water, followed by a mixture with $25 \mathrm{~mL}$ of the LNC-C suspension. Blank spray-dried lipid-core nanocapsule (SD-LNC-B) powders were prepared according to the same protocol, except curcumin was omitted from the original nanocapsule suspension. Three independent batches of SD-LNC-C and SD-LNC$\mathrm{B}$ were prepared, stored at room temperature and protected from light.

\section{Physicochemical Characterization of SD-LNC-C}

\section{Process Yield}

The process yield was determined as the ratio between the weight of the experimentally obtained solid mass and the sum of the weights of all other components, discounting the water content of the suspensions and added before the spraydrying step.

\section{Drug Content}

SD-LNC-C samples (0.695 g) were diluted in water $(5 \mathrm{~mL})$ and vortexed for $2 \mathrm{~min}$. A $1-\mathrm{mL}$ aliquot of this redispersion was diluted in acetonitrile $(10 \mathrm{~mL})$. These samples were centrifuged at $4120 \times g$ for $10 \mathrm{~min}$. Lastly, the supernatant was collected and diluted in mobile phase (1:5, $\mathrm{v} / \mathrm{v})$. The samples were filtered $(0.45-\mu \mathrm{m}$ PVDF membrane) and immediately analysed by the RP-HPLC method described in "Drug Content Analysis."

\section{Morphological Characterization of SD-LNC-C}

\section{Scanning Electron Microscopy}

The SD-LNC-C surface morphology was evaluated by scanning electron microscopy (SEM) (Jeol Scanning Microscope, JSM-6060, Tokyo, Japan) at $10 \mathrm{kV}$. The samples were observed at different magnifications (between $\times 1000$ and $\times 20,000)$. These analyses were carried out at the Centro de Microscopia e Microanalises of the Federal University of Rio Grande do Sul (UFRGS, Brazil). The samples were coated with gold before analysis (Jeol Jee 4B SVG-IN, Tokyo, Japan).

\section{Confocal Raman Microscopy}

To investigate the spatial component distribution in the SD-LNC-C structure, confocal Raman microscopy (CRM) measurements using a WITec alpha $300 \mathrm{R}^{+}$(WITec GmbH, Ulm, Germany) were performed. The excitation wavelength of the applied monochromatic laser was $532 \mathrm{~nm}$, adjusted to a power of $20 \mathrm{~mW}$. A confocal pinhole of $50-\mu \mathrm{m}$ rejected signals from out-of-focus regions, and the $\times 50$ magnification objective (Epiplan Neofluar, Zeiss, Germany) with a N.A. 0.8 was applied (19). Image pixels assigned to LNC-C were depicted in yellow, whereas drying adjuvant (maltodextrin and L-leucine) spectra were depicted in blue.

\section{Performance of the Aqueous SD-LNC-C Redispersion}

Aiming to evaluate the efficiency of the SD-LNC-C redispersion in contact with water, dispersion of the SD-LNCC samples $(0.695 \mathrm{~g})$ in water $(5 \mathrm{~mL})$ were carried out, using vortex mixture for $2 \mathrm{~min}$ at room temperature. This amount of powder $(0.695 \mathrm{~g})$ in $5 \mathrm{~mL}$ of water allowed the recovery of the initial curcumin concentration in the LNC suspension $\left(1 \mathrm{mg} \mathrm{mL}{ }^{-1}\right)$. Moreover, standardization of the vortex time was used to simulate an aqueous redispersion and agitation of the SD-LNC-C formulation prior to oral administration to the patient. Particle size analyses were carried out by LD and DLS using the same parameters and experimental conditions described in "Particle Size and Zeta Potential Analyses."

\section{In vitro Drug Release Studies}

The in vitro drug diffusion profiles of non-encapsulated curcumin (ethanolic solution) and the release profiles of LNC-C and SD-LNC-C were evaluated using the dialysis sac method. SD-LNC-C samples $(0.695 \mathrm{~g})$ were diluted in water $(5 \mathrm{~mL})$ and vortexed for $2 \mathrm{~min}$. The dialysis bag $(12 \mathrm{kDa})$, containing $2 \mathrm{~mL}$ of curcumin solution (non-encapsulated drug), LNC suspension (1 $\left.\mathrm{mg} \mathrm{mL}^{-1}\right)$ or SD-LN-C after aqueous redispersion $\left(1 \mathrm{mg} \mathrm{mL} L^{-1}\right)$, was immersed in the release medium (water/ethanol/polysorbate $80(80: 20: 1 \mathrm{v} / \mathrm{v})$ $(200 \mathrm{~mL})$ at $\left.37^{\circ} \mathrm{C}\right)(5)$. At predetermined time intervals, aliquots $(1 \mathrm{~mL})$ of the release medium were collected and replaced with fresh medium to guarantee the sink condition. The samples were diluted with mobile phase $(1: 1 \mathrm{v} / \mathrm{v})$ and filtered through a $0.45-\mu \mathrm{m}$ PVDF membrane (Millipore ${ }^{\circledR}$, Darmstadt, Germany), and curcumin was assayed by the RPHPLC according to the method previously defined in "Drug Content Analyses," with slight modifications. The injection volume was adjusted to $50 \mu \mathrm{L}$ to increase the method sensitivity. This RP-HPLC method was linear $(y=338091 x-$ 9583, $r=0.9999$ ) between 0.1 and $5 \mu \mathrm{g} \mathrm{mL}{ }^{-1}$, precise (RSD lower than $3.3 \%$ ) and specific. The diffusion and release profiles were analysed by mathematical models using the KinetDS 3.0 software (20). Moreover, the dissolution efficiency (DE) (\%), a model-independent description of the dissolution curve, was calculated using the same software. Curcumin diffusion and release profiles were modelled by a semi-empirical zero-order equation (Eq. 1) and a first-order equation (Eq. 2). The best correlation coefficient and the best Akaike information criterion (AIC) provided by the 
software, as well as the best graphical adjustment, were considered to select the fitted model.

$Q=k t+Q_{0}$

$C=\frac{1}{Q}=k t+\frac{1}{Q_{0}}$

where:

$Q$ amount (\%) of curcumin released at the time $t$

$Q_{0} \quad$ start value of $Q$

$k \quad$ constant

\section{In vitro Biological Evaluation}

Curcumin has known neuroprotective properties, and its anti-inflammatory activity has been associated with this effect in neurodegenerative diseases. Curcumin may exert its antiinflammatory activity by interacting with different molecular targets, including proinflammatory cytokines, protein kinases and other enzymes (21). Based on that, the anti-inflammatory activity of LNC-C was assessed by measuring the levels of proinflammatory cytokines released in lipopolysaccharide (LPS)-stimulated organotypic hippocampal slice cultures.

\section{Organotypic Hippocampal Slice Cultures}

Hippocampal slice cultures were prepared from male Wistar rats (6- to 8-days old). All procedures were approved by the local Ethics Committee on the Use of Animals (CEPUFRGS, protocol number 20005). The protocol described by Stoppini and colleagues (22) was followed to prepare slice cultures as interface cultures, with some modifications (2224). Organotypic cultures were maintained at $5 \% \mathrm{CO}_{2} / 95 \%$ $\mathrm{O}_{2}$ atmosphere and $37^{\circ} \mathrm{C}$ for 14 days. The culture medium was replaced every other day.

\section{Culture Treatments}

LPS (Sigma-Aldrich, São Paulo, Brazil) was solubilized at $1 \mathrm{mg} \mathrm{mL}^{-1}$ in sterile bi-distilled water. A stock concentration of $100 \mathrm{mM}$ of non-encapsulated curcumin in dimethyl sulfoxide (DMSO) was freshly prepared for each experiment. LNC-C were prepared as previously described. $0.695 \mathrm{~g}$ of SDLNC-C samples were redispersed in $5 \mathrm{~mL}$ of water, followed by vortexing for $2 \mathrm{~min}$. Non-encapsulated curcumin, LNC-C and SD-LNC-C, after aqueous redispersion, were directly diluted in culture medium to the desired concentration. The increase in neuroprotection following pretreatment with curcumin, before LPS exposure, was evaluated. Thus, on the 12th day, non-encapsulated curcumin $(5 \mu \mathrm{M})$, LNC-C $(5 \mu \mathrm{M})$ or SD-LNC-C $(5 \mu \mathrm{M})$ were added to the culture medium $1 \mathrm{~h}$ prior to the exposure of hippocampal slices to LPS $\left(100 \mu \mathrm{g} \mathrm{mL}^{-1}\right)$ for $48 \mathrm{~h}$. The control cells were treated with an identical amount of each vehicle: DMSO 0.05\%, LNC-B (blank lipid-core nanocapsules suspension: without any encapsulated drug) or SD-LNC-B (redispersed spray-dried formulation containing LNC-B). After $48 \mathrm{~h}$, the culture medium was withdrawn, frozen and stored at $-20^{\circ} \mathrm{C}$ for the analysis of cytokine levels.

\section{Determination of Cytokine Levels}

The levels of tumour necrosis factor-alpha (TNF- $\alpha)$ and interleukin 1- $\beta$ (IL-1 $\beta$ ) released into the culture medium were analysed using ELISA assay kits (R\&D Systems, Minneapolis, MN, USA) following the manufacturer's protocol. Cytokines levels were expressed as picograms of each cytokine per millilitre of culture medium $( \pm$ SEM).

\section{Statistical Analysis}

Formulations were prepared and analysed in triplicate. Characterization and release study data are expressed as the mean \pm standard deviation. Cytokine levels are expressed as mean \pm standard error of the mean (SEM). Statistical analyses at a significance level of $p \leq 0.05$ were performed using one-way analysis of variance (ANOVA) and Tukey test as a post hoc (GraphPad Prism, version 5.0.1, USA).

\section{RESULTS}

\section{Curcumin-Loaded Lipid-Core Nanocapsules}

LNC-C suspensions had a bright yellow aspect. LD analysis revealed a unimodal nanometric population with $\mathrm{D}[3,4]$ of $215 \pm 4 \mathrm{~nm}$ and a span value of $1.40 \pm 0.08$. The DLS analysis confirmed the mean nanometric particle size $(206 \pm 6 \mathrm{~nm})$ and low PDI $(0.1 \pm 0.1)$. The zeta potential was negative for all batches, with a mean value of $-5.7 \pm 1 \mathrm{mV}$. The EE was $99 \pm 0.1 \%$. The total drug content was $0.95 \pm$ $0.03 \mathrm{mg} \mathrm{mL} \mathrm{m}^{-1}$, corresponding to $95 \%$ of the expected concentration of $1.00 \mathrm{mg} \mathrm{mL}^{-1}$.

\section{Spray-Dried Curcumin-Loaded Lipid-Core Nanocapsule Powders}

The SD-LNC-C resulted in a bright yellow, homogeneous and fine powder. The process yield was $68 \pm 3 \%$. The curcumin content in the powder was $6.5 \pm 0.2 \mathrm{mg} \mathrm{g}^{-1}$, corresponding to $96 \pm 5 \%$ of the expected value. SD-LNC-C was formed by smooth, spherical-shaped particles with occasional invaginations, as observed by the SEM (Fig. 1a).

\section{Confocal Raman Microscopy Analysis}

As previously reported, blank LNC and the mixture of leucine/maltodextrin can be differentiated based on their unique Raman spectra (19). In the present study, the spatial component distribution within the SD-LNC-C was visualized. False-colour images based on the Raman spectra showed agglomerates in which the nanocapsules (depicted in yellow) attached to the leucine/maltodextrin mixture (depicted in blue, Fig. 2a). Besides the agglomerates, smaller spray-dried assemblies were detected for which false colour images with a higher magnification indicated nanocapsules on the surface (Fig. 2b). 

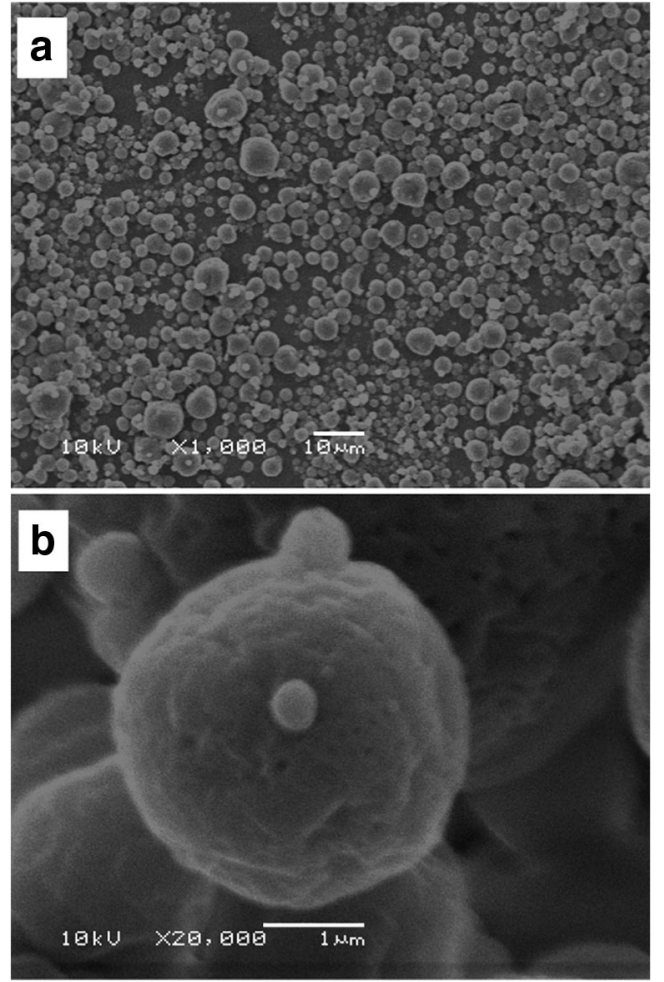

Fig. 1. SEM images of SD-LNC-C formulations a at $\times 1000$ magnification and $\mathbf{b}$ at $\times 20,000$ magnification

\section{Performance of the Aqueous SD-LNC-C Redispersion}

Particle size distribution profiles of LNC-C and the redispersed SD-LNC-C formulation obtained by LD analysis are shown in Fig. 3. Immediately after the redispersion, four major particle populations were observed (Fig. 3a). The profile of the particles whose size distribution was on the nanometre range was close to the profile of the original LNC$\mathrm{C}$ formulation. The particle size, expressed as a numberbased particle distribution (Fig. 3b), presented unimodal distribution, with a profile that was analogous to that of the original LNC-C suspension. Particle size distribution profiles of LNC-C and the redispersed SD-LNC-C formulation obtained by DLS analysis are shown as in the Supplementary Material (S1).

Moreover, Table I shows the mean size, PDI and $D$ values $(D 10, D 50$ and $D 90)$ of LNC-C and its respective
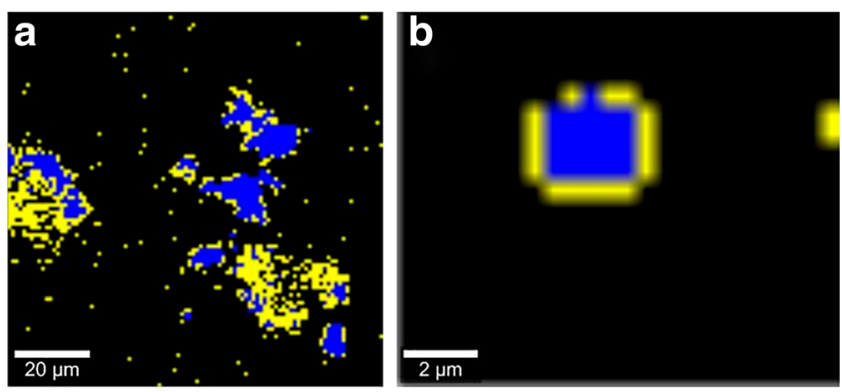

Fig. 2. False colour Raman images of spray-dried curcumin-loaded lipid-core nanocapsules (SD-LNC-C). a Agglomerates and b single spray-dried particle assembly. Maltodextrin and leucine are depicted in blue and LNC-C in yellow, respectively redispersed SD-LNC-C formulations, obtained by DLS and LD analysis.

\section{In vitro Drug Release Studies}

Figure 4 shows the in vitro release/diffusion profiles of curcumin from LNC-C, the redispersed SD-LNC-C and an ethanolic curcumin solution (non-encapsulated curcumin).

Curcumin diffusion from the ethanolic solution reached around $70 \%$ after $72 \mathrm{~h}$. In contrast, the percentage of curcumin released from LNC-C and the redispersed SDLNC-C reached around $25 \%$ and $34 \%$ after $72 \mathrm{~h}$, respectively. Table II shows the kinetic parameters regarding the zero-order kinetic model, which best described the drug release profiles for both formulations (LNC-C and SD-LNCC). In addition, the dissolution efficiencies (DE) were calculated, reaching $56.15 \pm 5.59 \%, 21.11 \pm 2.33 \%$ and 13.18 $\pm 0.64 \%$ for non-encapsulated curcumin, SD-LNC-C and LNC-C, respectively $(p<0.05)$.

\section{Effect of Curcumin on In vitro Neuroprotective Model}

Figure 5 shows the TNF- $\alpha$ and IL-1 $\beta$ levels of organotypic hippocampal cultures, with and without pretreatment with curcumin samples before exposure to LPS $\left(100 \mu \mathrm{g} \mathrm{mL}^{-1}\right)$ for $48 \mathrm{~h}$. TNF- $\alpha$ and IL-1 $\beta$ levels were higher in the medium after exposure to LPS in comparison with the
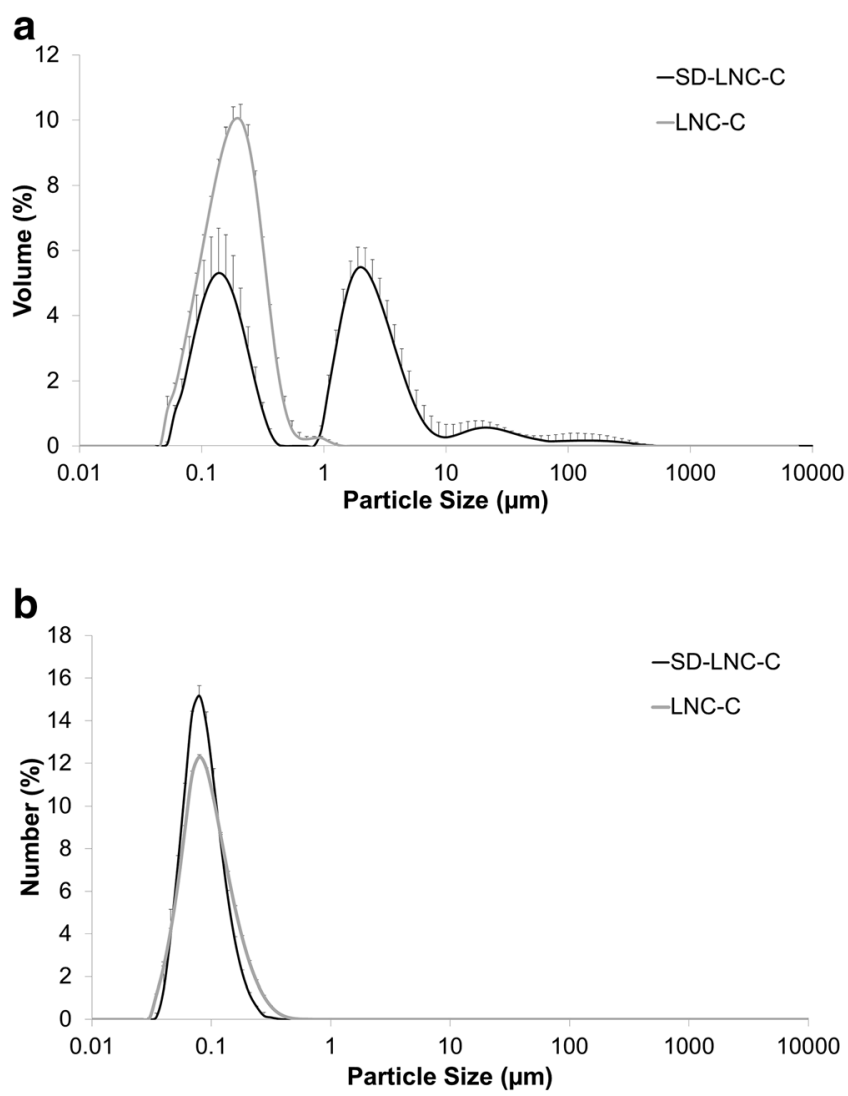

Fig. 3. Particle size distribution profile obtained by laser diffraction of LNC-C suspension and SD-LNC-C after aqueous redispersion ( $0.695 \mathrm{~g}$ in $5 \mathrm{~mL}$ of ultrapure water). a Particle size expressed as volume-based distributions and $\mathbf{b}$ particle size expressed as numberbased distributions $(n=3)$ 
Table I. Mean Size, Polydispersity Index (PDI) and $D$-Values ( $D 10$, $D 50$ and D90) of LNC-C and Redispersed SD-LNC-C Formulations $(n=4$ Independent Batches)

\begin{tabular}{lll}
\hline & LNC-C & SD-LNC-C \\
\hline$Z$-average (nm) & $206 \pm 03$ & $233 \pm 09$ \\
PDI & $0.10 \pm 0.01$ & $0.47 \pm 0.10$ \\
$D 10(\mathrm{~nm})$ & $131 \pm 02$ & $97 \pm 02$ \\
$D 50(\mathrm{~nm})$ & $218 \pm 04$ & $249 \pm 02$ \\
$D 90(\mathrm{~nm})$ & $378 \pm 20$ & $663 \pm 80$ \\
\hline
\end{tabular}

following control groups that were not exposed to LPS: DMSO $0.05 \%$, non-encapsulated curcumin, LNC-B, LNC-C, SD-LNC-B and SD-LNC-C. The mean proinflammatory cytokine levels of the control groups that were not exposed to LPS are not shown in Fig. 5, since they were below the detection limit of $0.125 \mathrm{pg} \mathrm{mL} \mathrm{m}^{-1}$. As observed in Fig. 5a, pretreatments with LNC-C and SD-LNC-C were able to attenuate the increase in TNF- $\alpha$ levels following LPS exposure for $48 \mathrm{~h}$ compared with the treatment with curcumin in its non-encapsulated form. Conversely, pretreatments with non-encapsulated curcumin, LNC-C or SD-LNC-C did not reduce IL-1 $\beta$ levels following LPS exposure (Fig. 5b).

\section{DISCUSSION}

LNC-C suspensions presented average diameters on the nanometre scale, a low PDI and negative zeta potential values, as expected due to their composition (polymer, oil and surfactants) and the preparation method used (5-7). The curcumin content and its encapsulation efficiency were close to $100 \%$. These results are consistent with previous data for this formulation (5).

Over the past few years, promising results have been reported for LNC-C, such as their ability to improve the protection of the CNS, after oral administration in rats, against a cognitive impairment and redox imbalance induced by cigarette smoke (7) and in a model of cognitive impairment induced by the peptide $A \beta(1-42)$ (6), as well as its

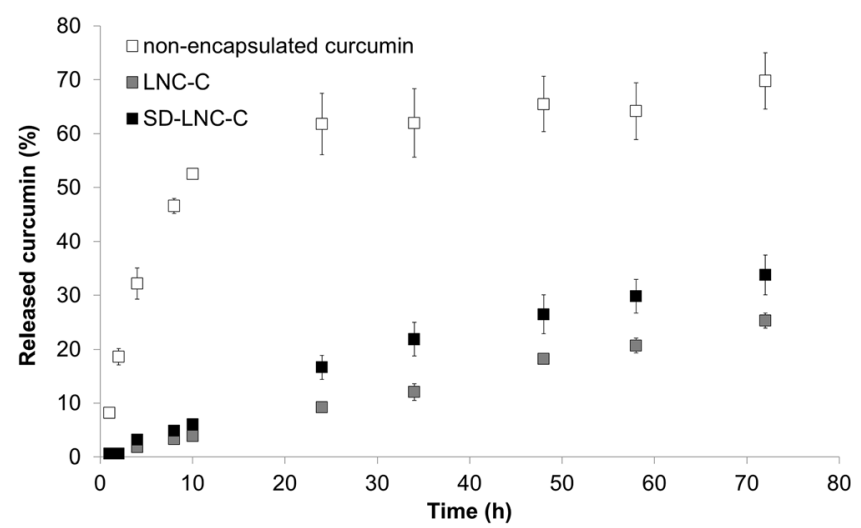

Fig. 4. In vitro curcumin release/diffusion profiles from curcuminloaded lipid-core nanocapsules (LNC-C), redispersed spray-dried curcumin-loaded lipid-core nanocapsules (SD-LNC-C) and ethanolic solution (non-encapsulated curcumin) after $72 \mathrm{~h}$
Table II. Curcumin Release Constants, Correlation Coefficients and AIC Obtained by Fitting the Release Profiles According to the ZeroOrder Equations

\begin{tabular}{lll}
\hline & LNC-C & SD-LNC-C \\
\hline$k\left(\% \mathrm{~h}^{-1}\right)$ & $0.35 \pm 0.02$ & $0.49 \pm 0.06$ \\
$r$ & $0.99 \pm 0.01$ & $0.97 \pm 0.01$ \\
AIC & $15.77 \pm 5.13$ & $41.47 \pm 5.88$ \\
\hline
\end{tabular}

Observed apparent kinetic constant $k$, correlation coefficient $(r)$ and Akaike information criterion (AIC) obtained by fitting curcumin release profiles to the zero-order equation. Results are expressed by mean $\pm S D, n=3$

increased bioavailability in the CNS in an in vivo glioma model (5).

However, despite these potentialities, the industrial application of LNC suspensions is limited due to problems associated with their long-term physicochemical and
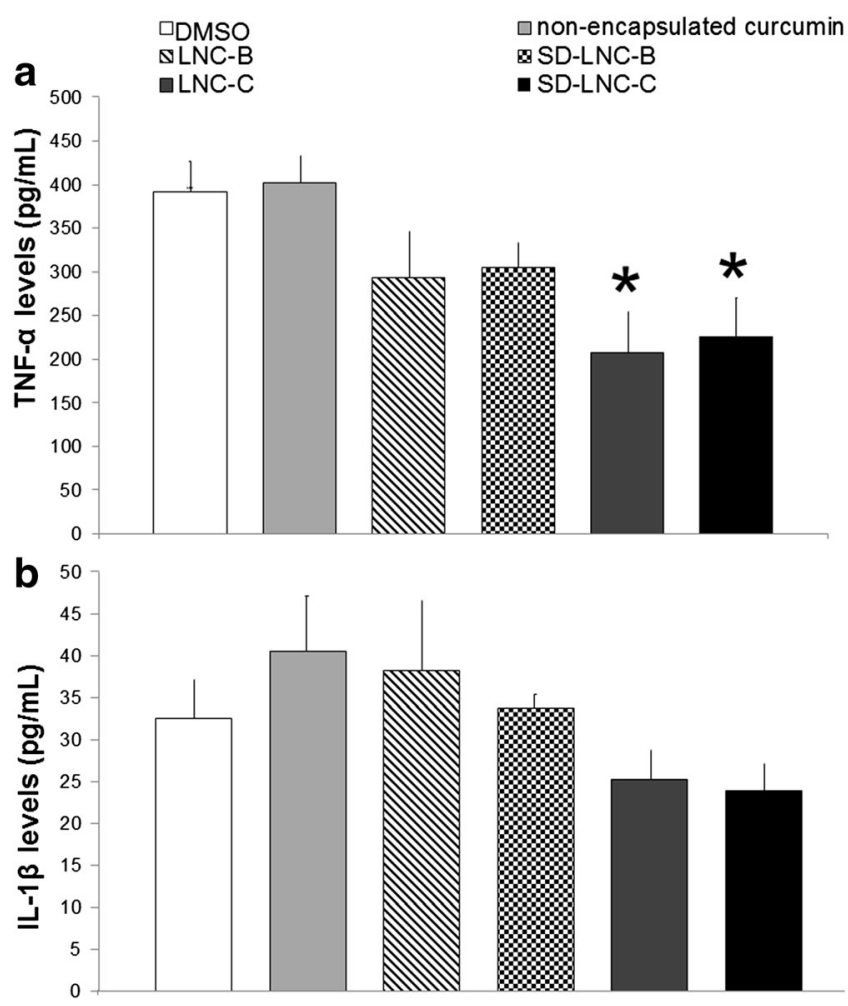

Fig. 5. LPS-induced increase in TNF- $\alpha$ and IL-1 $\beta$ levels in organotypic hippocampal cultures can be attenuated by LNC-C. a LNC-C and SD-LNC-C prevented the LPS-induced increase in TNF$\alpha$ in the culture medium after exposure of hippocampal slices to LPS. b Non-encapsulated curcumin, LNC-C and SD-LNC-C did not prevent the LPS-induced increase in IL-1 $\beta$ levels by LNC-C in the culture medium after exposure of hippocampal slices to LPS. Pretreatment with vehicle (DMSO $0.05 \%$ ), non-encapsulated curcumin $5 \mu \mathrm{M}$, LNC-C $5 \mu \mathrm{M}$, SD-LNC-C $5 \mu \mathrm{M}$, LNC-B or SDLNC-B $1 \mathrm{~h}$ before exposure to LPS for $48 \mathrm{~h}$. The measured TNF- $\alpha$ and IL-1 $\beta$ medium levels of control groups (without exposure to LPS) were below the method detection limit of $0.125 \mathrm{pg} \mathrm{mL}$. Results are expressed by mean \pm SEM, $n=5$. Statistically significant differences were denoted with asterisk when $p \leq 0.05$ (one-way ANOVA followed by Tukey's test post hoc) 
microbiological stability. To overcome this drawback, some technological strategies like freeze-drying, spray-drying and wet granulation have been proposed to convert polymeric nanocapsules into powders $(16-18,25,26)$. More recently, the formulation of redispersible spray-dried LNC was reported by our research group (19). However, in that previous study, there was no drug loaded in the LNC. Consequently, to highlight the potential use of this approach as a viable alternative for the development of novel oral dosage forms, it was fundamental to assess the effect of the spray-drying process on both physicochemical and biological properties of LNC loaded with an active substance. Hence, in the present study, LNC-C were produced to evaluate whether their neuroprotective properties would be affected by the drying process. Therefore, redispersible SD-LNC-C powders were produced and their physicochemical and biological behaviours were assessed. SD-LNC-C powders (3 batches) were produced with a mean yield of $68 \%$, which is in agreement with previous reports on the spray-drying of polymeric nanocapsules $(16,17)$. This powder formulation had a drug content close to $100 \%$ of the expected value, confirming that curcumin was not affected by heat or any other parameter of the process. This result agrees with the suitability of spraydrying to obtain microparticles containing curcumin, using a drying temperature of $150^{\circ} \mathrm{C}$ to produce microcapsules containing curcumin (27). Similarly, Machado et al. described that the spray-drying processes did not lead to great degradation of antioxidants present in the anthocyanin-rich extract from blackberry residues (28). In addition, SD-LNC-C was formed by smooth, spherical particles with occasional invaginations, in agreement with the particles described by Andrade et al. for powders prepared by spray-drying using LNC without drug loading (19). Their spherical shape has an important significance, considering that most unit operations to produce final solid dosage forms are based on the manipulation of particle sizes and transport of powders (milling, granulation, coating, sieving) (29). Therefore, the spherical shape of SD-LNC-C particles may improve the flow properties of the bulk powder, simplifying the transport operations during the manufacturing processes $(30,31)$.

According to Kho and colleagues, the aqueous redispersibility is highly dependent on the type and concentration ratios of the formulation constituents (32). Moreover, our previous report (19) showed that the complete coating of the drying adjuvants (maltodextrin and L-leucine) by the hydrophobic LNC could impair their aqueous redispersion. Thus, a chemically selective investigation using confocal Raman microscopy was carried out to analyse the component distribution of SD-LNC-C. Ideally, the maltodextrin/leucine mixture and the nanocapsules would form loose agglomerates in which water can easily intrude for homogeneous redispersion of the capsules after oral administration. Such agglomerates were successfully visualized in the present study. The blend of drying adjuvants (maltodextrin and Lleucine) form spacers between nanocapsules. In addition, the size of single spray-dried particle assemblies $(\sim 2 \mu \mathrm{m})$ agreed with the SEM investigations.

However, the suitable aqueous redispersion of the SDLNC-C has to be investigated as well as its drug release profile in comparison with LNC, since many biological advantages of nanocarriers are assigned to their physical and surface properties (particle size and surface area), in addition to their ability to control drug release. Dimer et al. (33) attributed a decrease in clearance and a long-lasting and pronounced effect of olanzapine-loaded LNC on the CNS, when compared with non-encapsulated drug, due to the polysorbate 80 coating of LNC. Moreover, Coradini et al. (34) described the improved antiedematogenic effect, in rats, of LNC containing co-encapsulated curcumin and resveratrol, when compared with the non-encapsulated polyphenols. These findings were explained based on the physicochemical characteristics of LNC, such as size, morphology and surface charge, which may influence the circulation and biodistribution of nanoparticles. For this reason, a rapid and easy aqueous redispersion of spray-dried nanocapsules, along with the protection of their integrity and drug release control properties, is fundamental for the success of these powder formulations.

SD-LNC-C had an adequate aqueous redispersion profile, recovering the particles in the nanometric range, with the nanoparticle size close to its size in the original suspension. Despite the higher PDI value, the $D 90$ value showed that the cumulative mass of $90 \%$ of the nanoparticles are below $1 \mu \mathrm{m}$ (Table I). These results are in agreement with a previous report (19) and showed that drug loading did not interfere in this redispersion behaviour. Moreover, it is important to note that, according to Andrade et al., the SEM analysis showed no changes in the morphological characteristics of the original nanoparticles after the spray-drying and redispersion steps. Regarding the in vitro drug release study, a controlled release profile was observed for both formulations (LNC-C and SDLNC-C), compared with the non-encapsulated curcumin, showing no influence of the spray-drying process on this property. This controlled drug release property is desirable to improve in vitro and in vivo curcumin effects, as previously reported (5,35-37). One of the goals of controlled drug release systems is to prolong the plasma concentration of a drug within its therapeutic window $(38,39)$. Additionally, the controlled release profiles showed by LNC-C and SD-LNC-C followed the zero-order kinetics, showing that both are able to provide a constant and sustained drug release, which is highly desirable. These systems spark great interest in the development of pharmaceutical forms, because they would release a drug at a predetermined rate for a specified period of time (40-42). However, a slight increase in the apparent release constant was calculated for SD-LNC-C, compared with the value obtained for $\mathrm{LNC}-\mathrm{C}$, showing that a slight rearrangement in the drug distribution in the particle may occur during the process used to convert the liquid suspension to the powder. A possible shift of the curcumin, strongly internalized, from the core of the nanocapsules to the polymeric wall during the dilution of LNC-C suspensions with water $(1: 1 \mathrm{v} / \mathrm{v})$, just before the drying step, may explain this finding. This shift would favour the release of curcumin in the medium, as previously reported for other drugs by Oliveira et al. (43). To estimate the amount of curcumin loss to the medium during this dilution step, the same protocol used to evaluate the encapsulation efficiency of the LNC-C suspension was performed with a sample containing the LNCC formulation diluted in ultrapure water at a ratio of 1:1 (v/v). Traces of curcumin could be observed in the chromatogram of the ultrafiltrate (data not shown), demonstrating a slight 
loss of curcumin to the medium in the dilution step. However, this concentration in the ultrafiltrate was below the limit of quantification of the analytical method $\left(5.0 \mu \mathrm{g} \mathrm{mL}{ }^{-1}\right)$. In contrast, this detection can be used to support our hypothesis, confirming that a slight shift of curcumin from the lipid core to the polymeric wall occurs during the dilution step before spray-drying, explaining the slight increase in the apparent release kinetic for SD-LNC-C.

To confirm these differences in the drug release data, the DE was calculated. This tool has important advantages to compare two or more pharmaceutical dosage forms. It represents the area under the dissolution curve up to a certain time, $t$, expressed as a percentage of a rectangle area representing the dissolution curve obtained by a hypothetic complete dissolution (100\%) up to the same time $t$. It enables the prompt comparison between formulations, and it can be theoretically associated with in vivo data $(44,45)$. DE values were different for the formulations evaluated, following the order: non-encapsulated curcumin > SD-LNC-C > LNC-C. The lowest DE values presented by SD-LNC-C and LNC-C compared with the curcumin ethanolic solution may be explained by the control of curcumin release promoted by its nanoencapsulation, as discussed above.

Overall, SD-LNC-C, and its redispersion in water, showed acceptable powder properties, nanoparticle recovery and a controlled drug release profile compared with the LNC$\mathrm{C}$ suspension. However, despite the adequate physicochemical properties of the LNC-C in the redispersed medium, it was fundamental to evaluate the effects of the drying and the aqueous redispersion processes on the biological activity of this formulation. This study was carried out using an in vitro neuroinflammation model as an in vitro screening test prior to in vivo studies.

Curcumin is known for its neuroprotective properties, and its anti-inflammatory activity has been linked to this effect in neurodegenerative diseases. Curcumin may exert its anti-inflammatory activity by interacting with different molecular targets, including proinflammatory cytokines, protein kinases and other enzymes (21). Neuroinflammatory responses include activation of microglia and astrocytes, playing an important role in modulating neurodegenerative processes. This inflammatory response results in the release of inflammatory mediators such as cytokines, chemokines, neurotransmitters and reactive oxygen species (46).

Based on that, the anti-inflammatory activities of nonencapsulated curcumin, LNC-C and redispersed SD-LNC-C were assessed based on the levels of proinflammatory cytokines released in LPS-stimulated organotypic hippocampal slice cultures. In addition, this protocol allows for identifying possible changes in the biological response promoted by curcumin nanoencapsulation, by the spraydrying technique and by the aqueous redispersion of the spray-dried powders.

Firstly, to evaluate whether LNC-C could improve the effectiveness of curcumin in protecting against neuroinflammation, the effect of the LNC-C suspension and redispersed SD-LNC-C, along with the non-encapsulated curcumin, in organotypic hippocampal cultures exposed to LPS was evaluated. These cultures have advantages over isolated cell cultures, such as the preservation of cellular interactions, making it possible to study the responses of neurons and glial cells $(47,48)$. According to Johansson and co-workers (49), LPS is widely accepted to induce inflammatory characteristics in hippocampal slices, which represents a useful in vitro model system for neuroinflammation and its link with studies on neurodegeneration processes. It is important to consider that this study evaluates the neuroprotective ability of LNC$\mathrm{C}$, considering the pretreatment of hippocampus slices against a neuroinflammatory stimulus, as a formulation to prevent the damage induced by LPS.

Both cytokines evaluated in this study, TNF- $\alpha$ and IL- $1 \beta$, exhibited higher release levels in the cells that persisted for at least $48 \mathrm{~h}$ after exposure to LPS compared with the controls not exposed to LPS. Higher concentrations of TNF- $\alpha$ released (on the order of 10 times) in relation to IL-1 $\beta$ were observed, which agreed with a previous report by Higashi et al. (50). The pretreatments with the LNC-C and SD-LNC$\mathrm{C}$ formulations were able to consistently block TNF- $\alpha$ release. However, no statistical differences in IL-1 $\beta$ release were observed considering the different pretreatments (LNC$\mathrm{C}$ and SD-LNC-C). As the IL- $1 \beta$ levels released in the samples were much lower in comparison with TNF- $\alpha$ levels, the IL-1 $\beta$ assay was performed close to the quantification limit of the ELISA $(0.125 \mathrm{pg} / \mathrm{mL})$, leading to high data dispersion, as shown by the SEM values in Fig. 4. These high SEM values may explain the non-statistical difference of the IL-1 $\beta$ levels between pretreatments with the different formulations.

Concerning TNF- $\alpha$ release levels, LNC-C improved the effects of curcumin against LPS compared with the nonencapsulated curcumin, reducing TNF- $\alpha$ levels. The sustained curcumin release from LNC could explain this difference. Consequently, lipid-core nanocapsules may promote the curcumin delivery to the cell in a controlled way, which allows a decrease in the level of cytokines released compared with the group treated only with LPS, even after $48 \mathrm{~h}$. In addition, the treatment with redispersed SD-LNC-C also reduced TNF- $\alpha$ levels in the cells compared with the treatment with non-encapsulated curcumin. No statistical differences in the TNF- $\alpha$ levels between the treatments with LNC-C and SD-LNC-C were found, showing that the drying step during the production of SD-LNC-C and the subsequent aqueous redispersion of the powder do not alter the in vitro biological activity of nanoencapsulated curcumin.

Taken together, the data obtained in the present study, using hippocampal slice cultures along with recent research about the versatility of the LNC and its ability to act on the CNS after oral administration in mice (13), encourage further studies on this subject in the effort to produce oral medicines composed of easily redispersible solid dosage forms containing SD-LNC-C. In the future, studies should be designed to evaluate the oral bioavailability of curcumin after oral administration of SD-LNC-C powders.

\section{CONCLUSIONS}

Curcumin-loaded lipid-core nanocapsules were formulated as redispersible spray-dried powders with no loss of their controlled drug release behaviour and their nanometric particle size distribution. Moreover, the spray-drying process did not affect the in vitro neuroprotective properties of nanoencapsulated curcumin against LPS-induced 
neuroinflammation in hippocampal slice cultures. This approach represents a significant contribution for the development of nanomedicines containing curcumin-loaded nanocarriers in the prevention and/or treatment of neurodegenerative diseases.

\section{ACKNOWLEDGEMENTS}

The authors gratefully receive the financial support of CAPES/Probral, CAPES (Finance Code 001), CNPq, FAPERGS, INCT_IF and the German Academic Exchange Service (DAAD). A PhD Fellowship was provided by CAPES-Brazil to D. F. de Andrade.

\section{COMPLIANCE WITH ETHICAL STANDARDS}

Conflict of Interest The authors declare that they have no conflict of interest.

\section{REFERENCES}

1. Gupta SC, Patchva S, Aggarwal BB. Therapeutic roles of curcumin: lessons learned from clinical trials. AAPS J. 2013;15(1):195-218. https://doi.org/10.1208/s12248-012-9432-8.

2. Belkacemi A, Doggui S, Dao L, Ramassamy C. Challenges associated with curcumin therapy in Alzheimer disease. Expert Rev Mol Med. 2011;13:e34. https://doi.org/10.1017/ S1462399411002055.

3. Shishodia S, Sethi G, Aggarwal BB. Curcumin: getting back to the roots. Ann N Y Acad Sci. 2005;1056:206-17. https://doi.org/ 10.1196/annals.1352.010.

4. Anand P, Kunnumakkara AB, Newman RA, Aggarwal BB. Bioavailability of curcumin: problems and promises. Mol Pharm. 2007;4(6):807-18. https://doi.org/10.1021/mp700113r.

5. Zanotto-Filho A, Coradini K, Braganhol E, Schroder R, de Oliveira CM, Simoes-Pires A, et al. Curcumin-loaded lipid-core nanocapsules as a strategy to improve pharmacological efficacy of curcumin in glioma treatment. Eur $\mathrm{J}$ Pharm Biopharm. 2013;83(2):156-67. https://doi.org/10.1016/j.ejpb.2012.10.019.

6. Hoppe JB, Coradini K, Frozza RL, Oliveira CM, Meneghetti $\mathrm{AB}$, Bernardi $\mathrm{A}$, et al. Free and nanoencapsulated curcumin suppress beta-amyloid-induced cognitive impairments in rats: involvement of BDNF and Akt/GSK-3beta signaling pathway. Neurobiol Learn Mem. 2013;106:134-44. https://doi.org/10.1016/ j.nlm.2013.08.001.

7. Jaques JA, Doleski PH, Castilhos LG, da Rosa MM, Souza V do C, Carvalho FB, et al. Free and nanoencapsulated curcumin prevents cigarette smoke-induced cognitive impairment and redox imbalance. Neurobiol Learn Mem. 2013;100:98-107. https://doi.org/10.1016/j.nlm.2012.12.007.

8. Venturini CG, Jager E, Oliveira CP, Bernardi A, Battastini AM, Guterres SR, et al. Formulation of lipid-core nanocapsules. Colloids Surf A Physicochem Eng Asp. 2011;375(1-3):200-8. https://doi.org/10.1016/j.colsurfa.2010.12.011.

9. Fiel LA, Rebêlo LM, Santiago TM, Adorne MD, Guterres SS, Sousa JS, et al. Diverse deformation properties of polymeric nanocapsules and lipid-core nanocapsules. Soft Matter. 2011;7(16):7240-7. https://doi.org/10.1039/C1SM05508A.

10. Ourique AF, Melero A, de Bona da Silva C, Schaefer UF, Pohlmann AR, Guterres SS, et al. Improved photostability and reduced skin permeation of tretinoin: development of a semisolid nanomedicine. Eur J Pharm Biopharm. 2011;79(1):95-101. https://doi.org/10.1016/j.ejpb.2011.03.008.
11. Frozza RL, Bernardi A, Paese K, Hoppe JB, da Silva T, Battastini AM, et al. Characterization of trans-resveratrolloaded lipid-core nanocapsules and tissue distribution studies in rats. J Biomed Nanotechnol. 2010;6(6):694-703. https:// doi.org/10.1166/jbn.2010.1161.

12. de Andrade DF, Fontana MC, Pohlmann AR, Guterres SS, Carlos R, Beck R. Nanoencapsulation of clobetasol propionate decreases its penetration to skin layers without changing its relative skin distribution. J Nanosci Nanotechnol. 2015;15(1):875-9. https://doi.org/10.1166/jnn.2015.9183.

13. Rodrigues SF, Fiel LA, Shimada AL, PN R, Guterres SS, Pohlmann AR, et al. Lipid-core nanocapsules act as a drug shuttle through the blood brain barrier and reduce glioblastoma after intravenous or oral administration. J Biomed Nanotechnol. 2016;12(5):986-1000. https://doi.org/10.1166/jbn.2016.2215.

14. Morishita M, Peppas NA. Is the oral route possible for peptide and protein drug delivery? Drug Discov Today. 2006;11:905-10. https://doi.org/10.1016/j.drudis.2006.08.005.

15. Schaffazick SR, Guterres SS, Pohlmann AR, Lucca FL. Caracterização e estabilidade físico-química de sistemas poliméricos nanoparticulados para administração de fármacos. Quim Nova. 2003;26(5):726-37. https://doi.org/10.1590/S010040422003000500017.

16. Hoffmeister CR, Durli TL, Schaffazick SR, Raffin RP, Bender EA, Beck RC, et al. Hydrogels containing redispersible spraydried melatonin-loaded nanocapsules: a formulation for transdermal-controlled delivery. Nanoscale Res Lett. 2012;7(1):1-13. https://doi.org/10.1186/1556-276X-7-251.

17. Marchiori MC, Ourique AF, da Silva Cde B, Raffin RP, Pohlmann AR, Guterres SS, et al. Spray-dried powders containing tretinoin-loaded engineered lipid-core nanocapsules: development and photostability study. J Nanosci Nanotechnol. 2012;12(3):2059-67. https://doi.org/10.1166/jnn.2012.5192.

18. Ourique AF, Chaves Pdos S, Souto GD, Pohlmann AR, Guterres SS, Beck RC. Redispersible liposomal-Nacetylcysteine powder for pulmonary administration: development, in vitro characterization and antioxidant activity. Eur $\mathbf{J}$ Pharm Sci. 2014;65:174-82. https://doi.org/10.1016/ j.ejps.2014.09.017.

19. Andrade DF, Vukosavljevic B, Benvenutti EV, Pohlmann AR, Guterres SS, Windbergs M, et al. Redispersible spray-dried lipid-core nanocapsules intended for oral delivery: the influence of the particle number on redispersibility. Pharm Dev Technol. 2018;23:414-25. https://doi.org/10.1080/10837450.2017.1400559.

20. Mendyk A, Jachowicz R, Fijorek K, Dorozynski P, Kulinowaki P, Polak S. KinetDS: an open source software for dissolution test data analysis. Dissolut Technol. 2012;19(1):6-11. https:// doi.org/10.14227/DT190112P6.

21. Aggarwal BB, Harikumar KB. Potential therapeutic effects of curcumin, the anti-inflammatory agent, against neurodegenerative, cardiovascular, pulmonary, metabolic, autoimmune and neoplastic diseases. Int J Biochem Cell Biol. 2009;41(1):40-59. https://doi.org/10.1016/j.biocel.2008.06.010.

22. Stoppini L, Buchs PA, Muller D. A simple method for organotypic cultures of nervous tissue. J Neurosci Methods. 1991;37(2):173-82. https://doi.org/10.1016/0165-0270(91)90128M.

23. Frozza RL, Horn AP, Hoppe JB, Simao F, Gerhardt D, Comiran RA, et al. A comparative study of beta-amyloid peptides Abeta1-42 and Abeta25-35 toxicity in organotypic hippocampal slice cultures. Neurochem Res. 2009;34(2):295303. https://doi.org/10.1007/s11064-008-9776-8.

24. Hoppe JB, Frozza RL, Horn AP, Comiran RA, Bernardi A, Campos MM, et al. Amyloid-beta neurotoxicity in organotypic culture is attenuated by melatonin: involvement of GSK-3beta, tau and neuroinflammation. J Pineal Res. 2010;48(3):230-8. https://doi.org/10.1111/j.1600-079X.2010.00747.x.

25. Friedrich RB, Fontana MC, Bastos MO, Pohlmann AR, Guterres SS, Beck RC. Drying polymeric drug-loaded nanocapsules: the wet granulation process as a promising approach. J Nanosci Nanotechnol. 2010;10(1):616-21. https:// doi.org/10.1166/jnn.2010.1732.

26. Abdewahed W, Degobert D, Stainmesse S, Fessi H. Freezedrying of nanoparticles: formulation, process and storage 
considerations. Adv Drug Deliv Rev. 2006;38(58):1688-713. https://doi.org/10.1016/j.addr.2006.09.017.

27. Liu W, Chen XD, Cheng Z, Selomulya C. On enhancing the solubility of curcumin by microencapsulation in whey protein isolate via spray drying. J Food Eng. 2016;169:189-95. https:// doi.org/10.1016/j.jfoodeng.2015.08.034.

28. Machado APF, Rezende CA, Rodrigues RA, Barbero GF, Rosa PTV, Martínez J. Encapsulation of anthocyanin-rich extract from blackberry residues by spray-drying, freeze-drying and supercritical antisolvent. Powder Technol. 2018;340:554-62. https://doi.org/10.1016/j.powtec.2018.09.063.

29. Hlinak AJ, Kuriyan K, Morris KR, Reklaitis GV. K. BP. Understanding critical material properties for solid dosage form design. J Pharm Innov. 2006;1:12-7. https://doi.org/10.1007/ BF02784876.

30. Hou H, Sun C. Quantifying effects of particulate properties on powder flow properties using a ring shear tester. J Pharm Sci. 2008;97(9):4030-9. https://doi.org/10.1002/jps.21288.

31. Fu X, Huck D, Makein L, Armstrong B, Willen U, Freeman T. Effect of particle shape and size on flow properties of lactose powders. Particuology. 2012;10:203-8. https://doi.org/10.1016/ j.partic.2011.11.003.

32. Kho K, Cheow WS, Lie RH, Hadinoto K. Aqueous redispersibility of spray-dried antibiotic-loaded polycaprolactone nanoparticle aggregates for inhaled anti-biofilm therapy. Powder Technol. 2010;203(3):432-9. https://doi.org/10.1016/ j.powtec.2010.06.003.

33. Dimer FA, Ortiz M, Pase CS, Roversi K, Friedrich RB, Pohlmann AR, et al. Nanoencapsulation of olanzapine increases its efficacy in antipsychotic treatment and reduces adverse effects. J Biomed Nanotechnol. 2014;10(6):1137-45. https:// doi.org/10.1166/jbn.2014.1817.

34. Coradini K, Friedrich RB, Fonseca FN, Vencato MS, Andrade DF, Oliveira CM, et al. A novel approach to arthritis treatment based on resveratrol and curcumin co-encapsulated in lipid-core nanocapsules: in vivo studies. Eur J Pharm Sci. 2015;78:163-70. https://doi.org/10.1016/j.ejps.2015.07.012.

35. Frozza RL, Bernardi A, Hoppe JB, Meneghetti AB, Matte A, Battastini AM, et al. Neuroprotective effects of resveratrol against Abeta administration in rats are improved by lipid-core nanocapsules. Mol Neurobiol. 2013;47(3):1066-80. https:// doi.org/10.1007/s12035-013-8401-2.

36. Fontana MC, Rezer JF, Coradini K, Leal DB, Beck RC. Improved efficacy in the treatment of contact dermatitis in rats by a dermatological nanomedicine containing clobetasol propionate. Eur J Pharm Biopharm. 2011;79(2):241-9. https://doi.org/ 10.1016/j.ejpb.2011.05.002.

37. Pohlmann AR, Fonseca FN, Paese K, Detoni CB, Coradini K, Beck RC, et al. Poly(-caprolactone) microcapsules and nanocapsules in drug delivery. Expert Opin Drug Deliv. 2013;10(5):623-38. https://doi.org/10.1517/ 17425247.2013 .769956$.

38. Zhao YN, Gu J, Jia S, Guan Y, Zhang Y. Zero-order release of polyphenolic drugs from dynamic, hydrogen-bonded LBL films.
Soft Matter. 2016;12(4):1085-92. https://doi.org/10.1039/ c5sm02186c.

39. Mody N, Tekade RK, Mehra NK, Chopdey P, Jain NK Dendrimer, liposomes, carbon nanotubes and PLGA nanoparticles: one platform assessment of drug delivery potential. AAPS PharmSciTech. 2014;15(2):388-99. https://doi.org/ 10.1208/s12249-014-0073-3.

40. Singhvi G, Singh M. Review: In vitro drug release characterization model. Int J Pharm Stud Res. 2011;2(1):77-84.

41. Gokhale A. Achieving zero-order release kinetics using multistep diffusion based drug delivery. Pharm Technol. 2014;38(5):1-3.

42. Wang L, Chen K, Wen H, Ouyang D, Li X, Gao Y, et al. Design and evaluation of hydrophilic matrix system containing polyethylene oxides for the zero-order controlled delivery of waterinsoluble drugs. AAPS PharmSciTech. 2017;18(1):82-92. https:// doi.org/10.1208/s12249-016-0498-y.

43. Oliveira CP, Venturini CG, Donida B, Poletto FS, Guterres SR, Pohlmann AR. An algorithm to determine the mechanism of drug distribution in lipid-core nanocapsule formulations. Soft Matter. 2012;9(4):1141-50. https://doi.org/10.1039/C2SM26959G.

44. Khan KA. The concept of dissolution efficiency. J Pharm Pharmacol. 1975;27(1):48-9. https://doi.org/10.1111/j.20427158.1975.tb09378.x.

45. Anderson NH, Bauer M, Boussac N, Khan-Malek R, Munden P, Sardaro M. An evaluation of fit factors and dissolution efficiency for the comparison of in vitro dissolution profiles. J Pharm Biomed Anal. 1998;17(4-5):811-22. https://doi.org/ 10.1016/S0731-7085(98)00011-9.

46. Tuppo EE, Arias HR. The role of inflammation in Alzheimer's disease. Int J Biochem Cell Biol. 2005;37(2):289-305. https:// doi.org/10.1016/j.biocel.2004.07.009.

47. Frotscher M, Zafirov S, Heimrich B. Development of identified neuronal types and of specific synaptic connections in slice cultures of rat hippocampus. Prog Neurobiol. 1995;45(6):viixxviii. https://doi.org/10.1016/0301-0082(95)90872-H.

48. Frozza RL, Bernardi A, Hoppe JB, Meneghetti AB, Battastini AM, Pohlmann AR, et al. Lipid-core nanocapsules improve the effects of resveratrol against Abeta-induced neuroinflammation. J Biomed Nanotechnol. 2013;9(12):2086-104. https://doi.org/ 10.1166/jbn.2013.1709.

49. Johansson S, Bohman S, Radesater AC, Oberg C, Luthman J. Salmonella lipopolysaccharide (LPS) mediated neurodegeneration in hippocampal slice cultures. Neurotox Res. 2005;8(34):207-20. https://doi.org/10.1007/BF03033974.

50. Higashi Y, Aratake T, Shimizu S, Shimizu T, Nakamura K, Tsuda M, et al. Influence of extracellular zinc on M1 microglial activation. Sci Rep. 2017;7:1-13. https://doi.org/10.1038/ srep43778.

Publisher's Note Springer Nature remains neutral with regard to jurisdictional claims in published maps and institutional affiliations. 